\title{
Substance Use Domain
}

National Cancer Institute

\section{Source}

National Cancer Institute. Substance Use Domain. NCI Thesaurus. Code C49615.

A subject domain utilized for the submission of information encompassing and representing data, vocabulary or records related to substance use. 